Gut and Liver, Vol. 13, No. 2, March 2019, pp. 197-205

\title{
Liver Cirrhosis, Not Antiviral Therapy, Predicts Clinical Outcome in Cohorts with Heterogeneous Hepatitis B Viral Status
}

\author{
Mi Na Kim ${ }^{1}$, Seong Gyu Hwang ${ }^{1}$, Beom Kyung Kim², Jun Yong Park², Do Young Kim², Kwang-Hyub Han², Seung Up Kim², \\ and Sang Hoon Ahn ${ }^{2}$ \\ ${ }^{1}$ Department of Internal Medicine, CHA Bundang Medical Center, CHA University School of Medicine, Seongnam, and ${ }^{2}$ Department of Internal \\ Medicine, Yonsei University College of Medicine, Seoul, Korea
}

Background/Aims: Antiviral therapy (AVT) reduces the risk of hepatocellular carcinoma (HCC) development in patients with chronic hepatitis $B(\mathrm{CHB})$. This multicenter retrospective study investigated the effects of AVT and hepatitis B virus (HBV)-related factors on the risk of HCC development in a cohort with heterogeneous HBV status. Methods: A total of 1,843 patients with $\mathrm{CHB}$ from two institutions were included in this study. Ultrasound and laboratory tests, including the $\alpha$-fetoprotein test, were conducted regularly to detect HCC development. Results: The mean age of our study population (1,063 men and 780 women) was 49.4 years. Cirrhosis was identified in 617 patients (33.5\%). During follow-up (median, 42.5 months), 81 patients developed HCC (1.39\% per person-year). A total of 645 patients (35.0\%) received ongoing AVT at enrollment. Ongoing AVT was not significantly associated with the risk of HCC development (all p>0.05). HBV-related variables (HBV DNA level, hepatitis B e antigen status, and alanine aminotransferase level) were also not significantly associated with the risk of HCC development (all $p>0.05)$. In contrast, cirrhosis was significantly associated with the risk of HCC development, regardless of adjustment (adjusted hazard ratio=4.098 to 7.020; all p<0.05). Cirrhosis significantly predicted the risk of HCC development in subgroups with and without ongoing AVT at enrollment, regardless of adjustment. Conclusions: Our study showed that cirrhosis, not AVT and HBV-related variables, was associated with HCC development in a cohort of patients with heterogeneous HBV status. Our results may help clinicians apply individualized surveillance strategies according to fibrotic status in patients with CHB. (Gut Liver 2019;13:197-205)

Key Words: Liver cirrhosis; Fibrosis; Antiviral therapy; Hepati- tis B; Clinical outcome

\section{INTRODUCTION}

As persistently high levels of hepatitis B virus (HBV) replication are closely associated with an increased risk for liver cirrhosis and hepatocellular carcinoma (HCC), ${ }^{1}$ replication-suppressing antiviral therapy (AVT) is the mainstay of current management for chronic hepatitis B (CHB)., ${ }^{2,3}$ This is strongly supported by a landmark randomized placebo-controlled trial by Liaw et al., ${ }^{4}$ which stratified CHB patients with advanced fibrosis or cirrhosis into lamivudine and placebo arms and found a significant benefit against HCC development in the lamivudine arm. Similarly, several subsequent meta-analyses have confirmed the beneficial influence of AVT in the long-term outcomes of CHB patients.

Due to this proven benefit of AVT against HCC development, no randomized placebo-controlled trials of drugs with a high genetic barrier, including entecavir or tenofovir, are available. Accordingly, recent studies have adopted cohorts of untreated historical controls to investigate the influence of drugs with a high genetic barrier on HCC development. Among several historical control-matched studies, ${ }^{7-10}$ a large, retrospective-prospective study by Wong et al. ${ }^{7}$ included 1,446 patients treated with entecavir and 424 untreated historical controls. In this study, entecavir significantly reduced the 5-year incidence rates of HCC (hazard ratio $[H R]=0.55)$, hepatic events (HR=0.51), liverrelated mortality $(\mathrm{HR}=0.26)$, and all-cause mortality $(\mathrm{HR}=0.34)$ in patients with liver cirrhosis but not in the entire patient population. $^{7}$

Although randomized, placebo-controlled trials and historical control-matched studies have shown that AVT reduces the risk of HCC development in CHB patients (particularly those with

Correspondence to: Seung up Kim ${ }^{a}$ (https://orcid.org/0000-0002-9658-8050) and Sang Hoon Ahn ${ }^{\text {b }}$

Department of Internal Medicine, Yonsei University College of Medicine, 50 Yonsei-ro, Seodaemun-gu, Seoul 03722, Korea

${ }^{\mathrm{a} T e l}$ ++82-2-2228-1982, Fax: +82-2-393-6884, E-mail: ksukorea@yuhs.ac

${ }^{b}$ Tel: +82-2-2228-1936, Fax: +82-2-393-6884, E-mail: ahnsh@yuhs.ac

Received on May 8, 2018. Revised on August 23, 2018. Accepted on August 24, 2018. Published online February 12, 2019

pISSN 1976-2283 eISSN 2005-1212 https://doi.org/10.5009/gnl18204

() This is an Open Access article distributed under the terms of the Creative Commons Attribution Non-Commercial License (http://creativecommons.org/licenses/by-nc/4.0) which permits unrestricted non-commercial use, distribution, and reproduction in any medium, provided the original work is properly cited. 
liver cirrhosis), the status of HBV infection is inevitably heterogeneous within $\mathrm{CHB}$ cohorts at any given time in the real-world setting. CHB patients with a high viral load but who are in the immune tolerant phase or with low viral load in the inactive carrier phase do not require AVT, whereas those in the immune clearance or reactivation phases require AVT to prevent disease progression. Thus, there is a possibility that the different clinical characteristics of each HBV status can potentially bias the influence of AVT on HCC development if untreated cohorts or historical controls are not available. Indeed, a recent study by Cho et al. ${ }^{11}$ showed that HCC risk remained higher in patients who even achieved complete virological remission than in patients with an inactive carrier status. ${ }^{11}$ Furthermore, another recent study by Park et al. ${ }^{12}$ showed that AVT was independently associated with a higher risk of HCC development due to the different baseline characteristics between $\mathrm{CHB}$ patients treated with AVT and those who did not receive AVT.

Thus, in this multicenter retrospective study, we investigated the influence of AVT as well as of HBV-related variables on the risk of HCC development in a cohort with heterogeneous HBV status.

\section{MATERIALS AND METHODS}

\section{Patients}

From January 2013 to December 2013, consecutive CHB patients from two institutions (Severance Hospital, Yonsei University and CHA Bundang Medical Center, CHA University) were considered eligible for this study. $\mathrm{CHB}$ was defined as the persistent presence of serum hepatitis B surface antigen (HBsAg) for more than 6 months. The exclusion criteria were as follows: current or past history of HCC at enrollment, HCC development within 6 months after enrollment, current or past history of hepatic decompensation at enrollment, Child-Pugh B and C class, coinfection with hepatitis $\mathrm{C}$ and/or human immunodeficiency virus, liver transplant status, significant alcohol consumption, and insufficient clinical and laboratory data (Supplementary Fig. 1).

This study was performed in accordance with the ethical guidelines of the 1975 Declaration of Helsinki and was approved by the Institutional Review Boards at Severance Hospital (2018-0764-001) and at CHA Bundang Medical Center (201804-050). Written informed consent from enrolled patients was waived due to the retrospective nature of this study.

\section{Baseline work-up and follow-up}

At baseline, patients provided a full medical history; underwent physical examination and routine blood chemistry; were assessed for hepatitis B e antigen (HBeAg) and its antibody, serum HBV DNA level, and $\alpha$-fetoprotein (AFP) levels, underwent ultrasonography, and were screened for HCC. If no evidence of HCC was detected, patients were followed up every 6 months with ultrasonography and laboratory work-up, including AFP to screen for HCC. HBsAg and HBeAg were measured using standard enzyme-linked immunosorbent assays (Abbott Laboratories, Chicago, IL, USA). HBV DNA levels were measured using a real-time polymerase chain reaction assay (Amplicor HBV Monitor Test; Roche Diagnostics, Basel, Switzerland; detection limit approximately $20 \mathrm{IU} / \mathrm{mL}$ ).

\section{Antiviral therapy}

During the study, AVT was initiated based on the treatment guidelines developed by the Korean Association for the Study of the Liver. ${ }^{13}$ In cases of virologic breakthrough (defined as $>1$ $\log 10 \mathrm{IU} / \mathrm{mL}$ increase in serum HBV DNA level from nadir on two consecutive tests) or genotypic mutations, rescue therapy was applied, if appropriate.

\section{Diagnosis of cirrhosis and HCC}

Cirrhosis was diagnosed based on liver histology, ultrasonographic findings or clinical signs of portal hypertension, such as the presence of ascites, esophageal or gastric varices, and hepatic encephalopathy. ${ }^{14}$ HCC was diagnosed based on radiologic findings from computed tomography or/and magnetic resonance imaging or based on histologic evidence, in accordance with the guideline of the American Association for the Study of Liver Diseases. $^{15}$

\section{Statistical analyses}

Data are expressed as the mean \pm standard deviation, median with range, or number (\%) as appropriate. Differences among continuous and categorical variables were examined for statistical significance using the Student t-test (or the Mann-Whitney U-test, if appropriate) and the chi-square test (or Fisher exact test, if appropriate), respectively. Person-years of follow-up were calculated from the date of study entry to either the onset of HCC or the last follow-up date.

Patients were censored at the time of HCC development or at the last follow-up. The incidence rate was calculated by dividing the number of patients with newly diagnosed HCC by the corresponding person-years. Cumulative HCC incidence rates were analyzed using the Kaplan-Meier method and compared with the log-rank test. To estimate independent predictors of HCC development, univariate and subsequent multivariate Cox proportional hazard regression analyses were performed. HRs and corresponding 95\% confidence intervals (CIs) were used where indicated. Various multivariate logistic regression analyses were applied to determine the independent associations among HBVrelated variables (HBV DNA level, HBeAg status, and alanine aminotransferase [ALT] level), cirrhosis, and the risk of HCC development.

All statistical analyses were performed using SPSS software version 18.0 (SPSS Inc., Chicago, IL, USA). A p-value <0.05 was considered to indicate statistical significance. 


\section{RESULTS}

\section{Baseline characteristics}

Among 4,850 patients with CHB from two centers $(2,442$ patients from Severance Hospital, Yonsei University and 2,408 patients from CHA Bundang Medical Center, CHA University) who were considered eligible for this study, 3,007 patients were excluded according to our exclusion criteria. Finally, 1,843 patients were selected for statistical analysis (804 patients from Severance Hospital and 1,039 patients from CHA Bundang Medical Center) (Supplementary Fig. 1).

The baseline characteristics of the study population at enrollment are summarized in Table 1 . The mean age of our study population (1,063 men and 780 women) was 49.4 years. Cirrhosis was identified in 617 patients (33.5\%), and 453 patients (24.6\%) were HBeAg positive. The mean HBV DNA and ALT levels were $2.8 \log 10 \mathrm{IU} / \mathrm{mL}$ and $44.4 \mathrm{IU} / \mathrm{L}$, respectively. A total of 645 patients (35.0\%) received ongoing AVT at enrollment, which included lamivudine $(n=62,9.6 \%)$, telbivudine $(n=23$, $3.6 \%)$, clevudine ( $\mathrm{n}=10,1.6 \%)$, adefovir $(\mathrm{n}=89,13.8 \%)$, entecavir $(\mathrm{n}=315,48.8 \%)$, tenofovir $(\mathrm{n}=44,6.8 \%)$, or a combination regimen $(n=102,15.8 \%)$.

\section{Follow-up and HCC development}

The median follow-up period of the entire study population was 42.5 months (range, 6.1 to 51.7 months), constituting a total of 5,845 person-years. The follow-up periods of the two institutions were statistically similar (median 41.6 months vs 43.1 months, $\mathrm{p}=0.438$ ). During follow-up, 81 patients (49 [60.5\%] from Severance Hospital and 32 [39.5\%] from CHA Bundang Medical Center) developed HCC (1.39\% per 1 person-year). The cumulative incidence rates of HCC at 1 and 3 years were $0.6 \%$ and $3.4 \%$, respectively. Of the 81 patients who developed HCC, 39 (48.1\%) received AVT at enrollment, and 35 (43.2\%) initiated AVT after enrollment. The remaining seven (8.7\%) patients without AVT during the study period experienced HCC development (two with minimally increased ALT levels [1-2x upper limit of normal] and five with liver cirrhosis, possibly from HBeAg-negative CHB phase).

\section{Comparisons of baseline characteristics according to AVT status}

To investigate the reason for the similar or even higher risk of HCC development in patients with ongoing AVT at enrollment when compared with those without AVT, we compared the baseline characteristics of these two groups (Table 2). Patients receiving AVT at enrollment were significantly older (mean 50.3 years vs 48.9 years) and had a higher incidence of diabetes mellitus (13.3\% vs $10.0 \%$ ) and cirrhosis (42.8\% vs 28.5\%), lower AFP level (mean $4.5 \mathrm{ng} / \mathrm{mL}$ vs $6.9 \mathrm{ng} / \mathrm{mL}$ ), lower HBV DNA level (mean $1.6 \log \mathrm{IU} / \mathrm{mL}$ vs $3.4 \log \mathrm{IU} / \mathrm{mL}$ ), lower aspartate aminotransferase (AST) level (mean 28.3 IU/L vs 42.2 IU/L), lower ALT level (mean 28.2 IU/L vs 53.1 IU/L), and lower platelet count (mean $163 \times 10^{9} / \mathrm{L}$ vs $179 \times 10^{9} / \mathrm{L}$ ) than patients not engaged in AVT at enrollment (all $\mathrm{p}<0.05$ ).

We also compared the baseline characteristics between patients who initiated AVT after enrollment and those naïve to AVT during the study period (Supplementary Table 1). Patients

Table 1. Baseline Characteristics

\begin{tabular}{|c|c|c|c|c|}
\hline Variable & $\underset{(n=1,843)}{\text { All }}$ & $\begin{array}{c}\text { Yonsei University } \\
(\mathrm{n}=804,43.6 \%)\end{array}$ & $\begin{array}{l}\text { Cha University } \\
(\mathrm{n}=1039,56.4 \%)\end{array}$ & p-value \\
\hline \multicolumn{5}{|l|}{ Demographic } \\
\hline Age, yr & $49.4 \pm 11.4$ & $52.4 \pm 10.7$ & $47.1 \pm 11.3$ & $<0.001$ \\
\hline Male sex & $1,063(57.7)$ & $443(55.1)$ & $620(59.7)$ & 0.051 \\
\hline Diabetes mellitus & $206(11.2)$ & $44(5.5)$ & $162(15.6)$ & $<0.001$ \\
\hline Cirrhosis & $617(33.5)$ & $379(47.1)$ & $238(22.9)$ & $<0.001$ \\
\hline \multicolumn{5}{|l|}{ Laboratory } \\
\hline$\alpha$-Fetoprotein, ng/mL & $6.1 \pm 22.4$ & $5.7 \pm 21.5$ & $6.3 \pm 23.1$ & 0.579 \\
\hline HBeAg positivity & $453(24.6)$ & $154(19.2)$ & $299(28.8)$ & $<0.001$ \\
\hline HBV DNA, $\log \mathrm{IU} / \mathrm{mL}$ & $2.8 \pm 2.1$ & $2.7 \pm 2.0$ & $2.9 \pm 2.2$ & 0.021 \\
\hline Aspartate aminotransferase, IU/L & $37.3 \pm 57.6$ & $33.7 \pm 43.7$ & $40.1 \pm 66.4$ & 0.013 \\
\hline Alanine aminotransferase, IU/L & $44.4 \pm 90.5$ & $39.2 \pm 70.1$ & $48.5 \pm 103.5$ & 0.022 \\
\hline Serum albumin, $\mathrm{g} / \mathrm{dL}$ & $4.4 \pm 0.4$ & $4.3 \pm 0.3$ & $4.5 \pm 0.4$ & $<0.001$ \\
\hline Total bilirubin, $\mathrm{mg} / \mathrm{dL}$ & $0.8 \pm 0.5$ & $0.9 \pm 0.5$ & $0.8 \pm 0.4$ & $<0.001$ \\
\hline Platelet count, $10^{9} / \mathrm{L}$ & $174 \pm 60$ & $168 \pm 64$ & $178 \pm 57$ & $<0.001$ \\
\hline Ongoing antiviral therapy & $645(35.0)$ & 278 (34.6) & 367 (35.3) & 0.768 \\
\hline
\end{tabular}

Data are presented as mean \pm SD or number $(\%)$. $\mathrm{HBeAg}$, hepatitis B e antigen; HBV, hepatitis B virus. 
Table 2. Comparison of Baseline Characteristics between Patients with and without Ongoing AVT at Enrollment

\begin{tabular}{|c|c|c|c|}
\hline Variable & $\begin{array}{l}\text { With ongoing AVT at enrollment } \\
\qquad(\mathrm{n}=645,35.0 \%)\end{array}$ & $\begin{array}{l}\text { Without ongoing AVT at enrollment } \\
\qquad(\mathrm{n}=1,198,65.0 \%)\end{array}$ & $\mathrm{p}$-value \\
\hline \multicolumn{4}{|l|}{ Demographic } \\
\hline Age, yr & $50.3 \pm 10.4$ & $48.9 \pm 11.8$ & 0.011 \\
\hline Male sex & $387(60.0)$ & $676(56.4)$ & 0.152 \\
\hline Diabetes mellitus & $86(13.3)$ & $120(10.0)$ & 0.036 \\
\hline Cirrhosis & $276(42.8)$ & $341(28.5)$ & $<0.001$ \\
\hline \multicolumn{4}{|l|}{ Laboratory } \\
\hline$\alpha$-Fetoprotein, ng/mL & $4.5 \pm 14.1$ & $6.9 \pm 25.8$ & 0.009 \\
\hline HBeAg positivity & $176(27.3)$ & $277(23.1)$ & 0.054 \\
\hline HBV DNA, log IU/mL & $1.6 \pm 1.0$ & $3.4 \pm 2.2$ & $<0.001$ \\
\hline Aspartate aminotransferase, IU/L & $28.3 \pm 26.8$ & $42.2 \pm 68.3$ & $<0.001$ \\
\hline Alanine aminotransferase, IU/L & $28.2 \pm 31.2$ & $53.1 \pm 109.0$ & $<0.001$ \\
\hline Serum albumin, $\mathrm{g} / \mathrm{dL}$ & $4.4 \pm 0.4$ & $4.4 \pm 0.3$ & 0.211 \\
\hline Total bilirubin, $\mathrm{mg} / \mathrm{dL}$ & $0.9 \pm 0.5$ & $0.8 \pm 0.4$ & 0.901 \\
\hline Platelet count, $10^{9} / \mathrm{L}$ & $163 \pm 57$ & $179 \pm 62$ & $<0.001$ \\
\hline
\end{tabular}

Data are presented as mean \pm SD or number (\%).

AVT, antiviral therapy; HBeAg, hepatitis B e antigen; HBV, hepatitis B virus.

who initiated AVT after enrollment were significantly more likely to have cirrhosis (42.0\% vs $19.1 \%$ ) and HBeAg positivity (41.5\% vs 10.3\%), higher AFP level (mean $10.5 \mathrm{ng} / \mathrm{mL}$ vs 4.4 $\mathrm{ng} / \mathrm{mL}$ ), higher HBV DNA level (mean $4.2 \log \mathrm{IU} / \mathrm{mL}$ vs $2.9 \log$ $\mathrm{IU} / \mathrm{mL}$ ), higher AST level (mean $62.0 \mathrm{IU} / \mathrm{L}$ vs $28.4 \mathrm{IU} / \mathrm{L}$ ), higher ALT level (mean $81.3 \mathrm{IU} / \mathrm{L}$ vs $33.6 \mathrm{IU} / \mathrm{L}$ ), lower serum albumin level (mean $4.3 \mathrm{~g} / \mathrm{dL}$ vs $4.4 \mathrm{~g} / \mathrm{dL}$ ), higher total bilirubin level (mean 0.9 vs 0.8 ), and lower platelet count (mean $162 \times 10^{9} / \mathrm{L}$ vs $192 \times 10^{9} / \mathrm{L}$ ) than patients naïve to AVT during the study period (all $\mathrm{p}<0.05$ ).

\section{Association between HBV-related variables and risk of HCC development}

The HRs of HCC development by HBV-related variables (HBV DNA level, HBeAg status, and ALT level) and differently adjusted models are summarized in Table 3. All three variables were not significantly associated with the risk of HCC development, regardless of adjustment (all p>0.05). When the study population was divided into two groups with and without ongoing AVT ( $n=645$ [35.0\%] and $n=1,198$ [65.0\%], respectively), there was also no significant association between HBV-related variables and the risk of HCC development in both subgroups (all p>0.05) (Table 3).

In the entire cohort, the cumulative HCC incidence rates were not significantly different according to HBV DNA level ( $p=0.821$ by log-rank test, HBV DNA $\leq 100 \mathrm{IU} / \mathrm{mL}[\mathrm{n}=986,53.5 \%]$ vs $101-10,000 \mathrm{IU} / \mathrm{mL}[\mathrm{n}=470,25.5 \%]$ vs $10,001-1,000,000 \mathrm{IU} / \mathrm{mL}$ $[\mathrm{n}=178,9.7 \%]$ vs $>1,000,000 \mathrm{IU} / \mathrm{mL}[\mathrm{n}=209,11.3 \%]$ ) (Supplementary Fig. 2A), HBeAg status ( $\mathrm{p}=0.947$ by log-rank test, positive $[n=453,24.6 \%]$ vs negative $[n=1,390,75.4 \%]$ ) (Supplementary Fig. $2 \mathrm{~B}$ ), and ALT level ( $\mathrm{p}=0.325$ by log-rank test, $\leq 40 \mathrm{IU} / \mathrm{L}$ [n=1,364, 74.0\%] vs 41-80 IU/L [n=338, 18.3\%] vs $81-400 \mathrm{IU} / \mathrm{L}$ $[\mathrm{n}=123,6.7 \%]$ vs $>400 \mathrm{IU} / \mathrm{L}[\mathrm{n}=18,1.0 \%]$ ) (Supplementary Fig. 2C).

\section{Association between ongoing AVT and risk of HCC devel- opment}

The HRs of HCC development by ongoing AVT and differently adjusted models are summarized in Table 4 . In the entire population, ongoing AVT did not show a significant association with the risk of HCC development after adjustments (all $\mathrm{p}>0.05$ ). The association between ongoing AVT and risk of HCC development was assessed in each institution (Supplementary Table 2), and ongoing AVT was not associated with the risk of HCC development at Severance Hospital ( $n=804$ [43.6\%]) (all p>0.05). Although ongoing AVT showed an independent positive association with a higher risk of HCC development in minimally adjusted models in the subgroup from CHA Bundang Medical Center ( $\mathrm{n}=1,039$ [56.4\%]) (HR=2.265 [95\% CI, 1.113 to 4.611] in model 1, which adjusted for age and gender, and $\mathrm{HR}=2.217$ [95\% CI, 1.089 to 4.514] in model 2, which adjusted for age, gender, and diabetes mellitus; all $\mathrm{p}<0.05)$, the significance was attenuated in further models that adjusted for more covariates. The cumulative incidence rates of HCC in patients with ongoing AVT (0.9\% at 1 year and $4.2 \%$ at 3 years) tended to be higher than those in patients without ongoing AVT (0.3\% at 1 year and $2.8 \%$ at 3 years), but these differences did not show any statistical significance ( $p=0.076$ by log-rank test) (Fig. 1).

\section{Association between cirrhosis and risk of HCC develop- ment}

The HRs of HCC development by cirrhosis and differently 


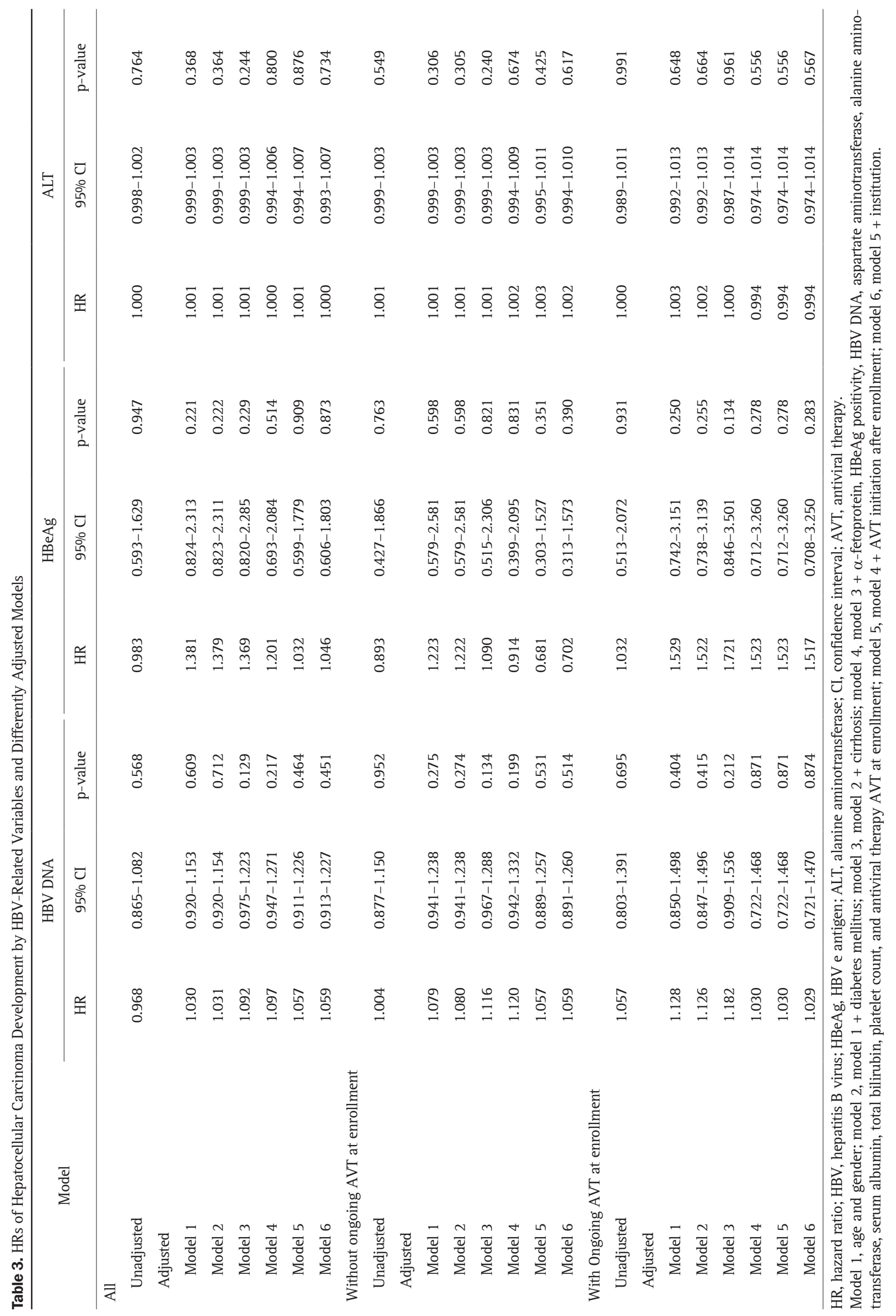


Table 4. HRs of Hepatocellular Carcinoma Development by Ongoing Antiviral Therapy and Differently Adjusted Models

\begin{tabular}{cccc}
\hline Model & HR & $95 \%$ CI & p-value \\
\hline Unadjusted & 1.480 & $0.957-2.291$ & 0.078 \\
Adjusted & & & \\
Model 1 & 1.475 & $0.952-2.283$ & 0.082 \\
Model 2 & 1.472 & $0.951-2.280$ & 0.083 \\
Model 3 & 1.141 & $0.735-1.771$ & 0.557 \\
Model 4 & 1.356 & $0.819-2.245$ & 0.236 \\
Model 5 & 1.352 & $0.817-2.237$ & 0.241 \\
\hline
\end{tabular}

$\mathrm{HR}$, hazard ratio; CI, confidence interval; $\mathrm{HBeAg}$, hepatitis B e antigen; HBV, hepatitis B virus.

Model 1, age and gender; model 2, model $1+$ diabetes mellitus; model 3, model $2+$ cirrhosis; model 4, model $3+\alpha$-fetoprotein, $\mathrm{HBeAg}$ positivity, HBV DNA, aspartate aminotransferase, alanine aminotransferase, serum albumin, total bilirubin, and platelet count; model 5 , model $4+$ institution.

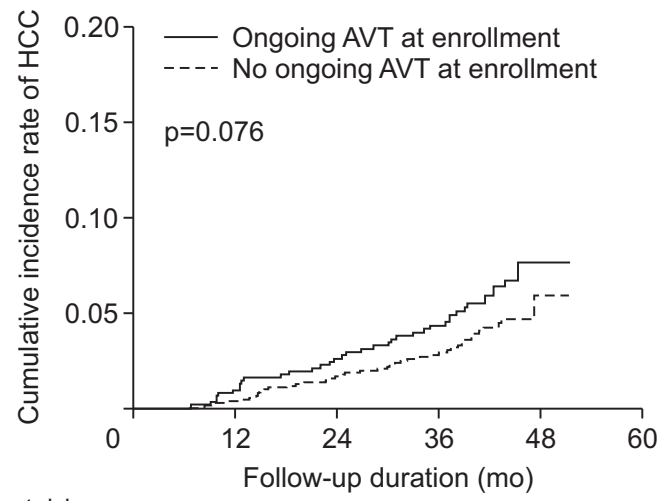

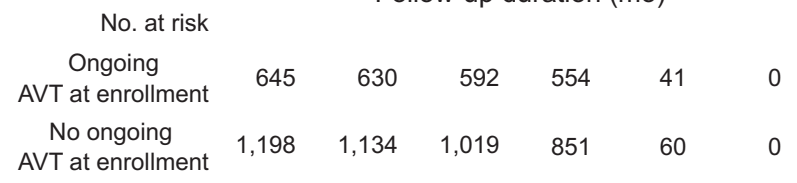

Fig. 1. Cumulative incidence rates of hepatocellular carcinoma (HCC) based on antiviral therapy (AVT) status at enrollment. The cumulative HCC incidence rates were not significantly different based on the AVT status at enrollment ( $\mathrm{p}=0.076$ by the log-rank test).

adjusted models are summarized in Table 5. In the entire population, cirrhosis showed a significant association with the risk of HCC development regardless of various adjustments (unadjusted $\mathrm{HR}=8.454$ [95\% CI, 4.824 to 14.813], adjusted $\mathrm{HR}=4.098$ to 7.020 ; all $\mathrm{p}<0.05)$. When the study population was stratified into two subgroups based on AVT status at enrollment, cirrhosis was a significant risk factor for HCC development in both subgroups, regardless of the adjustments (Supplementary Table 3).

The association between cirrhosis and risk of HCC development was assessed at each institution (Supplementary Table 4). Cirrhosis was significantly associated with the risk of HCC development at Severance Hospital regardless of adjustments (unadjusted HR=4.795 [95\% CI 2.327 to 9.883], adjusted HR=3.380 to 4.435 ; all $\mathrm{p}<0.05$ ), except for the borderline statistical sig-
Table 5. HRs of Hepatocellular Carcinoma Development by Cirrhosis and Differently Adjusted Models (All Study Participants)

\begin{tabular}{cccc}
\hline Model & HR & 95\% CI & p-value \\
\hline Unadjusted & 8.454 & $4.825-14.813$ & $<0.001$ \\
Adjusted & & & \\
Model 1 & 6.957 & $3.957-12.231$ & $<0.001$ \\
Model 2 & 7.020 & $3.992-12.347$ & $<0.001$ \\
Model 3 & 5.257 & $2.795-9.885$ & $<0.001$ \\
Model 4 & 5.117 & $2.754-9.733$ & $<0.001$ \\
Model 5 & 4.337 & $2.301-8.177$ & $<0.001$ \\
Model 6 & 4.095 & $2.148-7.807$ & $<0.001$ \\
\hline
\end{tabular}

$\mathrm{HR}$, hazard ratio; CI, confidence interval; $\mathrm{HBeAg}$, hepatitis B e antigen; HBV, hepatitis B virus; AVT, antiviral therapy.

Model 1, age and gender; model 2, model $1+$ diabetes mellitus; model 3 , model $3+\alpha$-fetoprotein, HBeAg positivity, HBV DNA, aspartate aminotransferase, alanine aminotransferase, serum albumin, total bilirubin, and platelet count; model 4, model $3+$ AVT at enrollment; model 5, model $4+$ AVT after enrollment; model 6, model $5+$ institution.

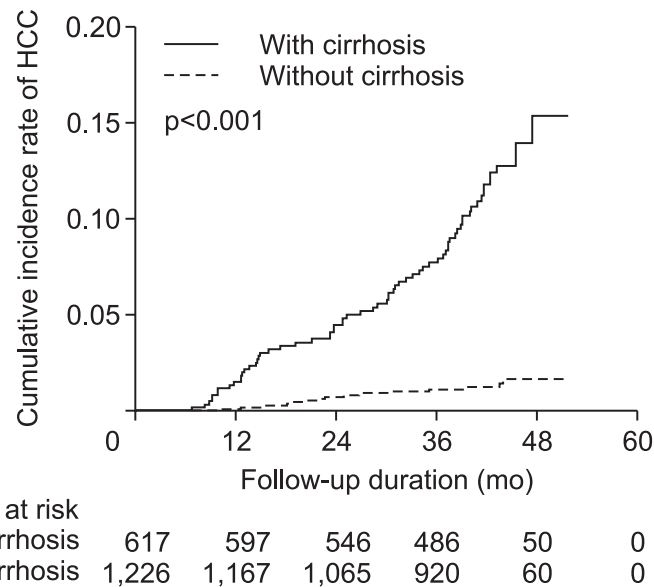

Fig. 2. Cumulative incidence rates of hepatocellular carcinoma (HCC) based on cirrhosis. The cumulative incidence rate of HCC in the subgroup with cirrhosis was significantly higher than that of the subgroup without cirrhosis ( $\mathrm{p}<0.001$ by the log-rank test).

nificance observed in model 5, which adjusted for age, gender, diabetes mellitus, laboratory tests, AVT at enrollment, and AVT initiation after enrollment ( $\mathrm{HR}=2.228$ [95\% CI, 0.966 to 5.141]; $\mathrm{p}=0.060$ ). In the subgroup from CHA Bundang Medical Center, cirrhosis was independently associated with the risk of HCC development regardless of adjustments (unadjusted $\mathrm{HR}=14.021$ [95\% CI, 5.770 to 34.070 ], adjusted $\mathrm{HR}=7.237$ to 11.347 ; all $\mathrm{p}<0.001$ ).

The cumulative incidence of HCC in the subgroup with cirrhosis was significantly higher than that of the subgroup without cirrhosis ( $p<0.001$ by log-rank test) (Fig. 2). The cumulative incidence rate of HCC in the subgroup with cirrhosis at 1 and 3 years was $1.5 \%$ and $7.6 \%$, respectively, whereas in the subgroup 
without cirrhosis, the rate was $0.1 \%$ and $1.1 \%$, respectively.

\section{DISCUSSION}

In this multicenter study comprising $\mathrm{CHB}$ patients with heterogeneous HBV status, AVT did not significantly predict HCC development after adjusting for other variables. In addition, HBVrelated variables such as HBV DNA, HBeAg, and ALT level were not associated with the risk of HCC development. In contrast, liver cirrhosis was significantly associated with the risk of HCC development regardless of adjustments (unadjusted HR=8.454, adjusted HR=4.098-7.020). Accordingly, the cumulative incidence of HCC in the subgroup with cirrhosis was significantly higher than that in the subgroup without cirrhosis.

Several studies have demonstrated a decrease in HCC development in patients with $\mathrm{CHB}$ who received AVT compared with historical controls who were unable to obtain AVT., ${ }^{4,8-20}$ However, in some previous studies that included patients receiving AVT and those in the inactive or immune tolerant phase who did not require AVT, AVT did not show a beneficial effect in preventing HCC development. A previous study ${ }^{21}$ that investigated predictors of HCC development in heterogeneous CHB cohorts showed that AVT was not significantly associated with HCC risk. By contrast, the degree of fibrotic burden, which was defined using transient elastography, was selected as an independent predictor of HCC development. In another study by Park et al., ${ }^{12}$ AVT was a significant predictor of HCC development, contrary to expectation, probably due to the unfavorable baseline characteristics of patients with AVT compared to those of patients without AVT; patients with AVT presented a higher body mass index, higher total bilirubin, higher AST level, lower serum albumin level, and higher degree of fibrotic burden than patients without AVT. We also investigated the influence of AVT on HCC development in a cohort with heterogeneous CHB status and found that, after adjusting for multiple variables, AVT showed no association with HCC development. More importantly, there was a trend of a higher risk of HCC development in patients receiving AVT at enrollment than in those not receiving AVT at enrollment.

Among 707 patients who did not receive AVT during the study period, seven patients developed HCC in our study. These patients who were not candidates for AVT according to Korean guidelines ${ }^{13}$ had minimally elevated ALT level or liver cirrhosis, possibly due to the HBeAg-negative $\mathrm{CHB}$ phase, indicating the importance of careful surveillance even in patients who do not undergo AVT. Regarding this issue, several studies showed that the indications of AVT should be expanded to individuals with lower serum HBV DNA levels and/or lower serum ALT levels. ${ }^{22,23}$ In addition, recent studies showed that HCC or progression to cirrhosis can develop even in patients in the immune tolerance or inactive carrier phases. ${ }^{24-26}$ However, no patients in the immune tolerance or inactive carrier phases developed HCC in our study, probably due to relatively small sample size of patients in these CHB phases in our study.

Because patients with worse baseline characteristics might have a higher chance of receiving AVT than those with favorable predictors such as low viral titer and normal ALT level, careful interpretation is needed regarding the influence of AVT when assessing mixed CHB cohorts. Indeed, in our cohort, compared with patients without AVT at enrollment, those with AVT had more confounding factors that could also be associated with an increased risk of HCC development, such as older age, higher incidence of cirrhosis, higher AFP level, higher total bilirubin level, and lower platelet level. The observed unfavorable characteristics in patients with AVT were quite similar to those reported by Park et al., ${ }^{12}$ as mentioned above. This phenomenon of inevitably imbalanced characteristics between patients with and without AVT might explain the varying influence of AVT on the risk of HCC development in heterogeneous HBV cohorts, which, in turn, suggests that cohorts with heterogeneous HBV status should be divided into subgroups with and without AVT and that the disease course of each cohort should be investigated separately.

In our study, we were able to clearly show that patients with cirrhosis had a higher risk for HCC than those without cirrhosis in a cohort with heterogeneous CHB status, regardless of AVT status. Several studies showed that appropriate AVT with potent antiviral agents cannot completely abolish the risk of HCC development. These studies also demonstrated that cirrhosis is a significant risk factor for HCC incidence during AVT. ${ }^{8,20,27-30}$ Moreover, several reports used recently developed noninvasive imaging and serum surrogates to assess the degree of liver fibrosis and showed that fibrotic burden is a significant risk factor for HCC development. ${ }^{31-37}$ A previous study by Kim et al., ${ }^{38}$ which used transient elastography to assess fibrotic burden, also reported a similar finding-the degree of liver fibrosis, rather than potent AVT with appropriate rescue therapy, was associated with the risk of HCC development. In another study by Kim et al., ${ }^{34}$ advanced fibrosis as defined by transient elastography was an important risk factor for HCC development, even in patients without clinical evidence of cirrhosis. They also showed that cirrhosis was an independent HCC risk factor in patients with and without AVT, which is consistent with the findings of our current study. Taken together, these data suggest that because patients with cirrhosis have a higher risk of HCC development regardless of AVT status, clinicians should actively assess the degree of liver fibrosis to develop an individual surveillance strategy for these patients.

In our study, well-known conventional risk factors of HCC development, such as HBV DNA level, HBeAg status, and ALT level, failed to show significant correlations with the risk of HCC development. Although these HBV-related variables have been useful in determining the disease state and in predicting clinical outcomes in patients with HBV infection until recently, this approach might be no longer appropriate in this era of ac- 
tive and potent AVT, especially when there is a need to predict clinical outcomes, including HCC development. Indeed, based on current guidelines, the higher risk of disease progression due to unfavorable characteristics such as high viral load and high ALT level can be significantly reduced due to the current potent AVTs, ${ }^{2,3,13,22}$ which suppress HBV DNA potently and rapidly normalize ALT. Therefore, it is not surprising that the influence of these conventional risk factors on the risk of HCC development was attenuated in our study.

We are also aware of several limitations of our study. First, our findings might have been influenced by the relatively short follow-up period (median, 42.5 months) because we tried to include patients treated with drugs with a high genetic barrier to reflect the current era of potent AVTs, including entecavir-based (48.8\%) and tenofovir-based (6.8\%) regimens, which were approved in Korea in 2007 and 2012, respectively. Thus, a subsequent study with a longer follow-up and with homogenous use of drugs with a high genetic barrier would be required. Second, the simple stratification of cirrhosis based on ultrasound might have biased our results. As is commonly known, the diagnostic accuracy of ultrasound for cirrhosis has been mixed. Indeed, a recent study has shown that compared to transient elastography, ultrasonographic evaluation can miss the diagnosis of early compensated cirrhosis. ${ }^{34}$ Thus, further studies that use more recent and accurate noninvasive imaging surrogates to assess the stepwise increase in fibrotic burden are required to validate our results. Third, more detailed information regarding fibrotic burden based on either histology or transient elastography was not available. However, because fibrotic burden can be variable even in patients undergoing AVT, future studies with fibrotic burden-matched cohorts that compare patients with and without AVT might provide more relevant clinical implications. Finally, the baseline characteristics of patients from two institutions were significantly different. Accordingly, the proportion of patients who developed HCC was significantly different between the institutions. However, we tried to reduce the potential bias by adjusting for institutions after adjusting HBV-related variables, AVT, and cirrhosis to estimate risk of HCC development. The overall results were similar between the two institutions, which might indicate the reproducibility of our results regardless of the baseline characteristics of the study population.

In conclusion, in our study, AVT as well as HBV-related variables, including HBV DNA level, HBeAg status, and ALT level, were unexpectedly not associated with HCC development. Thus, to prevent the biased influence of AVT on the risk of HCC development, it would be better to analyze the separated cohort based on AVT and not the heterogeneous CHB cohorts in future HBV studies. Furthermore, in our study, patients with cirrhosis had a higher risk of HCC development, indicating that cirrhosis, not AVT, can predict HCC development in cohorts with heterogeneous HBV status. Our results may help clinicians apply individualized surveillance strategies according to fibrotic status in patients with $\mathrm{CHB}$.

\section{CONFLICTS OF INTEREST}

No potential conflict of interest relevant to this article was reported.

\section{ACKNOWLEDGEMENTS}

This study was supported by the Basic Science Research Program through the National Research Foundation of Korea funded by the Ministry of Science, ICT \& Future Planning (2016R1A1A1A05005138), and the Ministry of Education, (2015R1D1A1A01058653). The funders had no role in the study design, data collection and analysis, decision to publish, or preparation of the manuscript. The authors are grateful to Dong-Su Jang, (Medical Illustrator, Medical Research Support Section, Yonsei University College of Medicine, Seoul, Republic of Korea) for his help with the figures.

Author contributions: Conception and design: S.U.K. and S.H.A.; development of methodology: S.U.K., S.H.A., and M.N.K.; acquisition, analysis and interpretation of data: all authors; writing, review, and/or revision of the manuscript: all authors; administrative, technical, or material support: M.N.K., S.U.K., and S.H.A.; study supervision: S.U.K. and S.H.A.

\section{REFERENCES}

1. Iloeje UH, Yang HI, Su J, et al. Predicting cirrhosis risk based on the level of circulating hepatitis B viral load. Gastroenterology 2006;130:678-686.

2. European Association for the Study of the Liver. Electronic address: easloffice@easloffice.eu; European Association for the Study of the Liver. EASL 2017 Clinical Practice Guidelines on the management of hepatitis B virus infection. J Hepatol 2017;67:370-398.

3. Liaw YF, Kao JH, Piratvisuth T, et al. Asian-Pacific consensus statement on the management of chronic hepatitis B: a 2012 update. Hepatol Int 2012;6:531-561.

4. Liaw YF, Sung JJ, Chow WC, et al. Lamivudine for patients with chronic hepatitis B and advanced liver disease. N Engl J Med 2004;351:1521-1531.

5. Papatheodoridis GV, Chan HL, Hansen BE, Janssen HL, Lampertico P. Risk of hepatocellular carcinoma in chronic hepatitis B: assessment and modification with current antiviral therapy. J Hepatol 2015;62:956-967.

6. Papatheodoridis GV, Lampertico P, Manolakopoulos S, Lok A. Incidence of hepatocellular carcinoma in chronic hepatitis B patients receiving nucleos(t)ide therapy: a systematic review. J Hepatol 2010;53:348-356.

7. Wong GL, Chan HL, Mak CW, et al. Entecavir treatment reduces hepatic events and deaths in chronic hepatitis B patients with liver cirrhosis. Hepatology 2013;58:1537-1547. 
8. Hosaka T, Suzuki F, Kobayashi M, et al. Long-term entecavir treatment reduces hepatocellular carcinoma incidence in patients with hepatitis B virus infection. Hepatology 2013;58:98-107.

9. Yasunaka T, Ikeda F, Wada N, et al. Entecavir reduces hepatocarcinogenesis in chronic hepatitis B patients. Acta Med Okayama 2016;70:1-12.

10. Kumada T, Toyoda H, Tada T, et al. Effect of nucleos(t)ide analogue therapy on hepatocarcinogenesis in chronic hepatitis B patients: a propensity score analysis. J Hepatol 2013;58:427-433.

11. Cho JY, Paik YH, Sohn W, et al. Patients with chronic hepatitis B treated with oral antiviral therapy retain a higher risk for HCC compared with patients with inactive stage disease. Gut 2014;63:1943-1950.

12. Park YE, Kim BK, Park JY, et al. Gamma-glutamyl transpeptidaseto-platelet ratio is an independent predictor of hepatitis B virusrelated liver cancer. J Gastroenterol Hepatol 2017;32:1221-1229.

13. Korean Association for the Study of the Liver. KASL clinical practice guidelines: management of chronic hepatitis B. Clin Mol Hepatol 2016;22:18-75.

14. Ahn J, Lim JK, Lee HM, et al. Lower observed hepatocellular carcinoma incidence in chronic hepatitis B patients treated with entecavir: results of the ENUMERATE study. Am J Gastroenterol 2016;111:1297-1304.

15. Bruix J, Sherman M; American Association for the Study of Liver Diseases. Management of hepatocellular carcinoma: an update. Hepatology 2011;53:1020-1022.

16. Yuen MF, Seto WK, Chow DH, et al. Long-term lamivudine therapy reduces the risk of long-term complications of chronic hepatitis B infection even in patients without advanced disease. Antivir Ther 2007;12:1295-1303.

17. Matsumoto A, Tanaka E, Rokuhara A, et al. Efficacy of lamivudine for preventing hepatocellular carcinoma in chronic hepatitis B: a multicenter retrospective study of 2795 patients. Hepatol Res 2005;32:173-184.

18. Eun JR, Lee HJ, Kim TN, Lee KS. Risk assessment for the development of hepatocellular carcinoma: according to on-treatment viral response during long-term lamivudine therapy in hepatitis B virus-related liver disease. J Hepatol 2010;53:118-125.

19. Papatheodoridis GV, Manolakopoulos S, Touloumi G, et al. Virological suppression does not prevent the development of hepatocellular carcinoma in HBeAg-negative chronic hepatitis B patients with cirrhosis receiving oral antiviral(s) starting with lamivudine monotherapy: results of the nationwide HEPNET. Greece cohort study. Gut 2011;60:1109-1116.

20. Kurokawa M, Hiramatsu N, Oze T, et al. Long-term effect of lamivudine treatment on the incidence of hepatocellular carcinoma in patients with hepatitis B virus infection. J Gastroenterol 2012;47:577-585.

21. Kim DY, Song KJ, Kim SU, et al. Transient elastography-based risk estimation of hepatitis B virus-related occurrence of hepatocellular carcinoma: development and validation of a predictive model. Onco Targets Ther 2013;6:1463-1469.
22. Lok AS, McMahon BJ. Chronic hepatitis B: update 2009. Hepatology 2009;50:661-662.

23. Lee KS, Kim DJ; Korean Association for the Study of the Liver Guideline Committee. Management of chronic hepatitis B. Korean J Hepatol 2007;13:447-488.

24. Chu CM, Liaw YF. Incidence and risk factors of progression to cirrhosis in inactive carriers of hepatitis B virus. Am J Gastroenterol 2009;104:1693-1699.

25. Chen JD, Yang HI, Iloeje UH, et al. Carriers of inactive hepatitis B virus are still at risk for hepatocellular carcinoma and liver-related death. Gastroenterology 2010;138:1747-1754.

26. Kim GA, Lim YS, Han S, et al. High risk of hepatocellular carcinoma and death in patients with immune-tolerant-phase chronic hepatitis B. Gut 2018;67:945-952.

27. Wang JP, Kao FY, Wu CY, et al. Nucleos(t)ide analogues associated with a reduced risk of hepatocellular carcinoma in hepatitis B patients: a population-based cohort study. Cancer 2015;121:1446-1455.

28. Orito E, Hasebe C, Kurosaki M, et al. Risk of hepatocellular carcinoma in cirrhotic hepatitis B virus patients during nucleoside/ nucleotide analog therapy. Hepatol Res 2015;45:872-879.

29. Papatheodoridis GV, Dalekos GN, Yurdaydin C, et al. Incidence and predictors of hepatocellular carcinoma in Caucasian chronic hepatitis B patients receiving entecavir or tenofovir. J Hepatol 2015;62:363-370.

30. Singal AK, Salameh H, Kuo YF, Fontana RJ. Meta-analysis: the impact of oral anti-viral agents on the incidence of hepatocellular carcinoma in chronic hepatitis B. Aliment Pharmacol Ther 2013;38:98-106.

31. Wong GL, Espinosa WZ, Wong VW. Personalized management of cirrhosis by non-invasive tests of liver fibrosis. Clin Mol Hepatol 2015;21:200-211.

32. Martin J, Khatri G, Gopal P, Singal AG. Accuracy of ultrasound and noninvasive markers of fibrosis to identify patients with cirrhosis. Dig Dis Sci 2015;60:1841-1847.

33. Martínez SM, Crespo G, Navasa M, Forns X. Noninvasive assessment of liver fibrosis. Hepatology 2011;53:325-335.

34. Kim MN, Kim SU, Kim BK, et al. Increased risk of hepatocellular carcinoma in chronic hepatitis B patients with transient elastography-defined subclinical cirrhosis. Hepatology 2015;61:1851-1859.

35. Seo YS, Kim MN, Kim SU, et al. Risk assessment of hepatocellular carcinoma using transient elastography vs. liver biopsy in chronic hepatitis B patients receiving antiviral therapy. Medicine (Baltimore) 2016;95:e2985.

36. Kim BK, Kim HS, Yoo EJ, et al. Risk assessment of clinical outcomes in Asian patients with chronic hepatitis B using enhanced liver fibrosis test. Hepatology 2014;60:1911-1919.

37. Suh B, Park S, Shin DW, et al. High liver fibrosis index FIB-4 is highly predictive of hepatocellular carcinoma in chronic hepatitis B carriers. Hepatology 2015;61:1261-1268.

38. Kim HS, Kim BK, Kim SU, et al. Association between level of fibrosis, rather than antiviral regimen, and outcomes of patients with chronic hepatitis B. Clin Gastroenterol Hepatol 2016;14:1647-1656. 